\title{
Jean-Marie Mayeur \\ Les catholiques français face au défi de
l'extrémisme politique
}

Dans une réflexion sur la démocratie française face à l'extrémisme politique entre les deux guerres mondiales, une interrogation sur l'attitude des catholiques est indispensable pour plusieurs raisons. La très grande majorité de la population française n'est-elle pas baptisée? Le taux de pratique malgré de vifs contrastes ne demeure-t-il pas appréciable? Les œuvres et organisations se réclamant du catholicisme n'ont-elles pas, de longue date, un poids considérable? L'essor de l'Action catholique générale, et spécialisée par milieu dans le cas des mouvements de jeunesse, n'est-il pas un trait marquant du catholicisme français depuis la fin des années 20 ? Mais un examen de l'attitude des catholiques français face aux tentations extrémistes n'est véritablement pertinent que s'il porte sur les catholiques pratiquants et, cercle plus restreint, sur les catholiques engagés à des niveaux divers dans la vie de l'Eglise. ${ }^{1}$ Il paraît clair qu'élargir la réflexion à l'ensemble de la population de tradition catholique, exprimée par le baptême et l'attachement aux grands rites de la pratique qui ponctuent les saisons de la vie, n'aurait pas de grande signification. En revanche, les prises de position des évêques et clercs, des laïcs, hommes d'œuvres, militants, engagés ou non dans les luttes politiques, doivent entrer dans le champ de l'analyse. Soucieux d'éviter une extension fallacieuse ou polémique, on considérera comme relevant de l'extrémisme les partis ou organisations qui contestent la démocratie libérale et envisagent contre elle le cas échéant le recours à la violence.

$\mathrm{Au}$ lendemain de la première guerre mondiale, la seule organisation proche du monde catholique qui relève de cette définition est la Ligue de l'Action française. Une idée volontiers reçue voudrait que les catholiques français au lendemain de la première guerre mondiale subissent de manière dominante l'influence de l'école de pensée néo-monarchiste de Charles Maurras. L'affirmation vaut sans doute pour une partie de l'épiscopat, des professeurs de séminaire et une élite intellectuelle laïque, tous séduits par la philosophie politique de Maurras, sa condamnation du libéralisme et de la démocratie issus de la Révolution française. Mais le comportement politique des catholiques ainsi aux élections dites du Bloc national de 1919 les porte bien plus vers la droite conservatrice et les modérés que vers les néomonarchistes. Faut-il redire que les indépendants, seul groupe proche des idées maurrassiennes, sont moins de trente dans la Chambre de 1919? Leurs professions de foi se terminent par "Vive la France ", sans mention de la République, et sont avant tout une mise en cause de la politique laïque et un appel au renforcement de l'exécutif. Elles traduisent, notamment dans l'Ouest, la persistance d'une extrême

1 Renvoyons une fois pour toutes à la présentation la plus approfondie qui offre une riche bibliographie: Gérard Cholvy et Yves-Marie Hilaire, Histoire religieuse de la France contemporaine, t. 2 et 3, Toulouse 1986 et 1988. 
droite traditionaliste. Ces députés ne sont pas élus sur les listes du Bloc national qui associent républicains modérés de tradition laïque et catholiques conservateurs ralliés à la République, mais sur des listes qui s'intitulent d'Action française et d'Union nationale. Certes, les catholiques intransigeants, tel l'historien Jean Guiraud dans $\mathrm{La}$ Croix ${ }^{2}$, sont favorables à ces listes et critiquent l'entente électorale avec les Républicains modérés au sein du Bloc national. Mais leur poids n'est pas déterminant. Les années de l'immédiat après-guerre sont pour les catholiques français celles du «second ralliement ». ${ }^{3}$ Il est à bien des égards la suite de «l'Union sacrée» et de la victoire de la République en 1918, il va dans le sens des orientations romaines ${ }^{4}$ et mène à l'établissement de bonnes relations entre Rome et la République.

Mais les orientations anticléricales du cartel des gauches, victorieux aux élections de 1924, mettent en cause cette situation. Elles suscitent de vives réactions de l'ensemble de l'opinion catholique et paraissent justifier les thèses des catholiques intransigeants. L'écho de l'Action française s'en trouve accru. Ses amis sont présents au sein de la Fédération nationale catholique du général de Castelnau fondée pour organiser la protestation face au cartel - organisation qui trouve un écho considérable. 5 Dans ce climat de lutte, l'Assemblée des cardinaux et archevêques publie le 10 mars 1925 une déclaration sur les lois dites de laïcité et sur les mesures à prendre pour les combattre. Elle a été préparée par un dominicain, thomiste, proche de l'Action française, le Père Janvier, adjoint de Mgr Chollet, archevêque de Cambrai à la commission permanente de l'Assemblée des cardinaux et archevêques ${ }^{6}$. Elle affirme avec une extrême vigueur l'hostilité aux lois laiques: «il ne nous est pas permis de leur obéir, nous avons le droit et le devoir de les combattre et d'en exiger, par tous les moyens honnêtes, l'abrogation ». Elle récuse la tactique qui «consisterait à ne pas heurter de front les législateurs laïcs» et invite à agir sur l'opinion et les législateurs en suivant l'exemple des socialistes, communistes et syndicalistes qui recourent à la manifestation et à la grève. N'était-ce pas un appel à la guerre au pouvoir en place, la justification des thèses de l'extrême droite?

La déclaration suscita en fait de telles réactions que le cardinal Dubois, archevêque de Paris, fit une mise au point en chaire, assurant qu'elle n'était pas une «déclaration de guerre», qu'elle n'était pas «un acte d'ordre politique», «l'Eglise reste au dessus et en dehors de la politique. Elle ne condamne ni ne patronne aucune forme légitime de gouvernement ". Le cardinal protestait contre l'accusation de

2 Qui déplore l'exclusion des royalistes, La Croix, 18 octobre 1919.

3 La formule est employée dès 1919, cf. Charles Pichon, L'Ame française du 6 septembre, commentant la lettre du cardinal Gasparri ci-dessous, et non, comme on le croit parfois, dans la décennie suivante.

4 Le cardinal Gasparri, secrétaire d'Etat de Benoit XV, dans la réponse qu'il adresse le 27 juillet 1919 au cardinal Luçon en réponse à la lettre collective de l'épiscopat français se félicite de ce que les évêques «donnent fort à propos aux catholiques de France le conseil de s'unir entre eux et avec d'autres citoyens de bonne volonté en suivant les directives pontificales, données par le passé et jamais révoquées", La Croix, 2 septembre 1919. C'est le rappel des orientations de Léon XIII.

5 A la fois groupe de pression, organe de défense religieuse, organisation d'Action catholique, elle aurait compté au moins un million de membres, cf. la thèse encore inédite de Corinne Bonafoux-Verrax, La Fédération nationale catholique 1924-1944, IEP Paris, 1999; compte rendu de soutenance par Xavier Boniface dans: Revue d'Histoire de l'Eglise de France, Janvier-Juin 1999, pp. 215-217.

6 Cf. André Laudouze, Dominicains français et Action française 1899-1940 - Maurras au couvent, Paris 1989, p. 76. 
vouloir renverser la République et présentait la déclaration comme «une thèse de théologie morale». Manifestement inspirée par Rome, cette mise au point marquait la volonté d'éviter toute apparence de collusion avec les ennemis du régime. Cette orientation fut facilitée par l'échec du cartel des gauches, mettant fin à ce qui paraissait un retour au temps du combisme.

Dans les années suivantes, la condamnation de l'Action française à la fin de 19267 , le renouvellement de l'épiscopat au long des années 30, le climat de paix religieuse et le déclin de l'anticléricalisme eurent pour conséquence d'enlever à l'extrême droite catholique tout soutien de la hiérarchie. Désormais s'affirme l'essor des mouvements d'Action catholique et du catholicisme social démocratique, dont les idées marquent non seulement le petit Parti démocrate populaire né à la fin de 1924, mais une partie de la droite modérée. Certes, les catholiques sont unanimes à demander une solution aux problèmes de l'enseignement privé, à la situation des congrégations, le plus souvent sous un simple régime de tolérance. On ne saurait considérer que ces revendications suffisent à définir quelque extrémisme. Dans la même période l'écho des Ligues anti-parlementaires est modeste. Ce sont les dernières années, entre 1926 et 1932, de fonctionnement satisfaisant de la République parlementaire.

La montée des périls extérieurs à partir de 1933, les progrès de l'anti-parlementarisme devant la crise du régime et la menace du communisme enfin, conduisirent une frange de l'opinion catholique à juger que les événements donnaient raison aux analyses de l'Action française ${ }^{8}$. D'autres se tournèrent vers les Ligues antiparlementaires ${ }^{9}$. Cette évolution est inséparable de la «radicalisation» des modérés qui caractérise la période. L'épiscopat fut attentif au risque de voir l'Eglise compromise. Il suffit de se reporter aux résolutions de l'Assemblée des cardinaux et archevêques de Mars 1936 à la veille des élections ${ }^{10}$. Le clergé «ne doit pas négliger de faire son devoir civique, mais il évitera soigneusement de s'inféoder aux partis politiques». Même consigne est adressée aux fidèles. Ils doivent avoir le «souci constant de maintenir l'Eglise et l'Action catholique en dehors et au dessus des partis ". C'est pourquoi «les dirigeants et militants d'Action catholique ne seront pas en même temps directeurs, représentants ou propagandistes d'un parti politique». A cet impératif de non-engagement politique est associée l'exigence de civisme: «il pratiqueront loyalement les vertus du citoyen et notamment le respect du pouvoir établi».

Après les partis, sont abordées les Ligues. Il est remarquable, et c'est le signe de son audience dans le monde catholique, que la Ligue des Croix de Feu soit nom-

7 On ne revient pas sur la condamnation romaine, son application, sa portée. Qu'il suffise de renvoyer à la thèse de Jacques Prévotat, Catholiques français et Action française - Etude de deux condamnations romaines, Université de Paris-Nanterre, 1994. Elle vient d'être publiée, Paris 2001. Les raisons de la condamnation sont certes d'abord religieuses et portent sur le «néo-positivisme» et le "politique d'abord" cher à l'école maurrassienne, mais le nationalisme exclusif et l'hostilité au régime de l'A.F. ne sont pas étrangers à la condamnation.

8 Laudouze, Dominicains français, pp. 250-254, donne à cet égard un rapport du P. Boisselot de la vie intellectuelle de l'été 1934 après les émeutes du 6 février qui insiste sur la "contagion» à nouveau du mouvement d'Action française.

9 La bibliographie sur le sujet est considérable, on se borne à renvoyer à l'Histoire des droites, dirigée par Jean François Sirinelli, 3 tomes, Paris 1992.

10 Publiées dans la Documentation catholique, 4-11 juillet 1936. 
mément visée: les membres du clergé inscrits au groupe des Croix de Feu au titre d'anciens combattants "s'abstiendront de prendre une part active à la vie de la Ligue». Plus généralement les prêtres s'abstiendront à l'avenir de donner leur nom à une Ligue politique, qu'elle qu'elle soit. Est réitérée enfin pour tous les catholiques l'invite à s'abstenir d'adhérer «à toute organisation qui serait en collusion avec un mouvement formellement condamné (allusion à l'Action française), qui se signalerait par une absence de principes chrétiens, qui s'appuierait sur des doctrines de violence ou d'irrespect vis à vis de l'Eglise». La mise en garde est nette.

De toutes les Ligues la Ligue des Croix de Feu fut, de loin, la plus importante. C'est elle surtout qui put attirer le plus grand nombre de catholiques. Elle se distingua des autres organisations par son caractère légaliste, et c'est pourquoi, si l'Assemblée des cardinaux et archevêques invite les prêtres à la réserve, rien de tel n'est signifié aux laïcs. Enfin, sans être confessionnelle, 11 l'organisation du colonel de la Rocque par son programme social et familial doit beaucoup aux orientations majeures du catholicisme social. On touche là à une des raisons de son écho dans le monde catholique ${ }^{12}$, séduit par un programme qui affirme la volonté de restaurer l'autorité de l'Etat sans mettre en cause la République et se réclame de l'idéal «travail, famille, patrie». En fait, ni les Croix de feu ni le Parti social français, fondé après la dissolution de la Ligue, ne peuvent être considérés comme des organisations extrémistes, même si leurs adversaires de gauche ont vu les choses ainsi à l'époque, et si ce mythe a eu la vie dure. Il suffit de voir la brochure de propagande du PSF où la France incarnée par Marianne avec le bonnet phrygien, représentation symbolique de la République si longtemps honnie de la droite catholique, s'oppose aux faisceaux, à la croix gammée, à la faucille et au marteau ${ }^{13}$. La volonté de réformer l'Etat et de remédier aux faiblesses de la démocratie parlementaire signifie certes la rupture avec une certaine conception de la République qui ne fut jamais celle de tous les Républicains ${ }^{14}$, mais non quelque extrémisme. La violence des polémiques de l'Extrême droite contre les Croix de Feu ${ }^{15}$, puis le PSF, est éclairante à cet égard.

S'il fallait isoler au sein du monde catholique des courants et des personnalités tentés par l'extrémisme politique dans les années 30, sans doute peut-on les chercher au sein de certains intellectuels de la «Jeune Droite» issue de l'Action française, et dans une partie de la Fédération républicaine. Après la condamnation de Maurras, des jeunes catholiques s'éloignèrent de lui et se retrouvèrent dans les re-

11 C'est pourquoi le terme de nationalisme chrétien qu'emploie Jacques Nobécourt dans son livre au reste remarquable (Le colonel de La Rocque 1885-1946 - ou les pièges du nationalisme chrétien, Paris 1996) n'est pas vraiment pertinent. Il n'est pas sûr non plus que le mot de nationalisme soit judicieux.

12 L'Hébdomadaire Sept du 28 décembre 1934 consacre un interview d'un ton sympathique au colonel de La Rocque; la Vie intellectuelle du 10 février 1935 rend compte de façon favorable de son livre Service public; cf. René Rémond, Les catholiques dans la France des années trente, Paris 1979, p. 131-132.

13 "Parti social français. Une mystique, un programme." L'illustration est à la page 4 de couverture, suivie de l'inscription «Ni fascisme, ni communisme! La France heureuse, forte et libre par le Parti social français".

14 C'est pourquoi la notion de «modèle républicain» n'est pas pleinement satisfaisante. Dès les débuts du régime, un certain nombre de membres de la famille républicaine déplorent l'évolution qui mène à la souveraineté absolue du parlement.

15 Leur comportement le 6 février 1934 où ils se bornent à une manifestation pacifique est dénoncé. 
vues des «non-conformistes» des années 3016 . Vivement critiques de la démocratie formelle et du parlementarisme, ils furent séduits par les expériences d'Etat autoritaire chrétien social, qu'il s'agisse du Portugal de Salazar ou de l'Autriche de Dollfuss. L'itinéraire d'un Jean de Fabrègues, fondateur de Réaction (1930-1932), cofondateur de Combat (1936-1939), est à cet égard remarquable ${ }^{17}$. Encore faut-il observer que ces petits cercles intellectuels qui ne sont pas spécifiquement catholiques ont un écho limité, et que leur discours qui se veut révolutionnaire ne s'accompagne guère en revanche d'un projet politique.

Plus significative est l'attitude des personnalités catholiques liées à la Fédération nationale catholique du général de Castelnau. Elles appartiennent à la Fédération républicaine dont une composante s'oriente vers des positions nettement hostiles au régime dans les années 3018 . Tel est le cas de Philippe Henriot, député de la Gironde en 1932, vice-président de la Fédération républicaine, et de Xavier Vallat, député de l'Ardèche. Ils sont liés à la Ligue anti-judéo-maçonnique que dirige un prélat intégriste, Mgr Jouin. Xavier Vallat, proche de l'Action française, incarne le traditionalisme maurrassien et rêve pour la France d'un régime qui s'inspire des idées corporatives du catholicisme social contre-révolutionnaire ${ }^{19}$ et se défende de l'influence des juifs ${ }^{20}$. D'autres représentants de l'extrême droite catholique insistent sur ces thèmes ${ }^{21}$. Philippe Henriot ${ }^{22}$ s'oriente nettement vers des formules autoritaires. La publication qu'il crée en avril 1934, La Jeunesse, organe de la Jeunesse de la Fédération républicaine de Gironde, exalte Salazar, Franco, Mussolini. S'amorce chez lui l'évolution qui va faire de l'ancien orateur de la Fédération nationale catholique un ultra de la collaboration, secrétaire d'Etat à l'Information et à la Propagande de Laval à partir du 1er janvier 1944. L'itinéraire de Philippe Henriot illustre une dérive possible dont on pourrait donner d'autres exemples ${ }^{23}$. Mais cette dérive est extrêmement minoritaire. Très rares sont les catholiques français acquis à des formules dictatoriales ou fascisantes.

Il importe ici d'éviter des amalgames. L'hostilité à la laïcité de l'Etat et de l'école, l'idéal corporatiste, la défense de la famille, tous ces thèmes autour desquels se retrouvent nombre de catholiques conservateurs ne suffisent pas à eux seuls à définir

16 Renvoyons au livre qui le premier a évoqué ce monde: Jean-Louis Loubet del Bayle, Les non-conformistes des années 30 - Une tentative de renouvellement de la pensée politique française, Paris 1969.

17 Récemment étudié par Véronique Chavagnac-Auzepy, Jean de Fabrègues (thèse de l'IEP, 1993), et Nicolas Kessler, Histoire politique de la Jeune Droite 1929-1942 (thèse de l'Université de ParisSorbonne, 1999). Ce travail vient d'être publiée, Paris 2001.

18 Ainsi de la Fédération républicaine du Rhône avec Victor Perret, cf. Mathias Bernard, La dérive des modérés. La Fédération républicaine du Rhône sous la Troisième République, Paris 1998.

19 Cf. Xavier Vallat, La Croix, les lys et la peine des hommes, Aymon 1960, où il évoque l'héritage légitimiste du catholicisme social.

20 On sait que Xavier Vallat, tenant de «l'antisémitisme d'Etat", fut Commissaire général aux questions juives de mars 1941 à mai 1942.

21 Henri de la Ferronnays et Jacques de Juigné, élus de la Loire-Inférieure, dans leur profession de foi pour les élections de 1936 se prononcent pour l'organisation professionnelle qui permettra à la France d'éviter la dictature fasciste et la révolution communiste, cité par Hélène et René Bourreau, Les députés parlent aux électeurs - Les professions de foi en Loire inférieure 1881-1936, dans: Monarchie et République, Publications de la Sorbonne, Paris 1999.

22 Sur lui la notice de Jacqueline Herpin, Dictionnaire des parlementaires d'Aquitaine sous la Troisième République, Bordeaux 1998, pp. 262-264.

23 On pense notamment au milicien Paul Touvier, venu de la jeunesse catholique de Haute-Savoie. 
l'extrémisme politique, même si l'extrême droite a pu les faire siens. Le refus du libéralisme et de la démocratie, le recours éventuel à la violence, la xénophobie et l'antisémitisme ${ }^{24}$ sont des critères plus pertinents. Encore faut-il observer, avec Richard Millman, que la xénophobie présente chez les Croix de Feu ${ }^{25}$ ne s'accompagne pas le plus souvent de racisme antisémite que désavoue La Rocque. Face à la crise des années 30 , la méfiance vis à vis des étrangers et des juifs récemment immigrés, sinon des juifs «français», s'étend au reste bien au delà des frontières de l'Extrême droite. L'Eglise catholique en revanche a pris position avec fermeté à la fin des années 30 face aux persécutions antisémites et au racisme. Qu'il suffise de citer la lettre du cardinal Verdier, archevêque de Paris, contre le racisme après la «nuit de cristal»: «des milliers et des milliers d'hommes sont traqués comme des bêtes fauves", que Dieu «écarte de nous ce racisme que répudie notre civilisation chrétienne»26. Dans sa conférence au théâtre Marigny, le 20 janvier 1939, le même cardinal Verdier affirme: "notre pays uni aux grandes démocraties apparait au monde comme le défenseur de la liberté des peuples». La presse, les revues et bulletins liées à l'Eglise font un écho profond à ces prises de positions comme à celles d'autres membres de l'épiscopat français ou étranger et du pape lui-même.

Un regard sur l'attitude des catholiques français dans les années de l'entredeux-guerres convainc que ceux-ci pour le plus grand nombre, à la suite de l'Union sacrée, puis de la condamnation de l'Action française, acceptent le régime et la République. La différence avec les premières décennies du régime est manifeste. Certes, le statut des congrégations religieuses, victimes des lois du début du siècle, et la situation de l'école privée font l'objet de revendications pressantes. Demeure aussi l'idéal d'un Etat qui rendrait un culte à Dieu et ne serait pas athée ${ }^{27}$. Mais l'épiscopat n'en est pas moins acquis a l'oeuvre de redressement national d'Edouard Daladier à partir d'avril 1938 au nom de la défense des libertés et des droits de la personne. Il espère que la nouvelle politique qui s'engage ouvre la voie à un redressement moral. Démarche exceptionnelle, le 22 mars 1939 une lettre du cardinal Verdier au nom de l'Assemblée des cardinaux et archevêques au président du conseil assure celui-ci de leur soutien, affirme que la mission de la France est de «sauvegarder la vraie liberté, l'égalité foncière de tous les hommes et la fraternité chrétienne». ${ }^{28}$ Contrairement à ce qu'on pourrait penser, la faveur que les évêques français ont porté, pendant un temps, au Maréchal Pétain et à la Révolution nationale ne contredit pas ces analyses. Au delà du désarroi commun à la grande majorité des Français et du respect du pouvoir établi y comptent l'harmonie entre le

24 Renvoyons au livre de Richard Millman, La question juive entre les deux guerres - Ligues de droite et antisémitisme en France, Paris 1992, qui constitue une mise au point nuancée sur l'attitude des diverses Ligues.

25 «Si le problème ethnique ne se pose pas», disait le colonel de la Rocque, «la question des étrangers s'impose."

26 Cette lettre est publiée dans le Petit Journal, l'organe du PSF, le 22 novembre 1938, cf. Millman, La question juive, p. 257.

27 Le directeur de La Croix, le P. Merklen, dont la nomination après la crise de l'Action française fait évoluer le quotidien assomptionniste vers la démocratie d'inspiration chrétienne, s'en prend aux conceptions politiques issues de la Révolution française lors du cent-cinquantennaire de celle-ci, La Croix, 11 mai 1939: «Elles ont pactisé étroitement avec le libéralisme doctrinal, l'indifférentisme religieux, le laïcisme.»

$28 \quad$ La Croix, 1 avril 1939. 
discours moral, social et familial de Vichy et celui de l'Eglise, et la conviction que le régime de Vichy évitera que ne s'impose l'idéologie nazie qu'il est différent de l'extrémisme de la collaboration.

Au long des années 30 , les responsables et les militants des organisations catholiques, des mouvements d'Action catholique, générale et spécialisée, paraissent dans l'ensemble ne plus mettre au premier plan la défense religieuse. Mais ils n'en jugent que plus nécessaire la formation sociale et civique. Celle-ci peut conduire à des engagements politiques qui ont en commun de refuser les extrêmes de droite ou de gauche. Un publication comme Terre nouvelle, «organe des chrétiens révolutionnaires", fait l'objet d'une mise en garde par le conseil de vigilance du diocèse de Paris publiée le 15 février 1936 et d'une condamnation de l'Index en juillet 193629. C'est la nébuleuse démocratie d'inspiration chrétienne qui exerce le plus d'attrait aux côtés de la droite modérée ${ }^{30}$ éloignée des Ligues anti-parlementaires. Au sein des catholiques tentés par l'extrême droite, il est possible de distinguer, même s'il y a des passages de l'un à l'autre, les traditionalistes, réactionnaires au sens propre du terme, et les fascisants. Sans doute les premiers représentent-ils le groupe le plus important sous l'influence de l'Action française, tandis que les seconds paraissent passablement marginaux. Mais les uns et les autres sont à contrecourant de l'évolution des forces vives du catholicisme français au long de l'entredeux-guerres. On peut avancer l'hypothèse que l'enseignement des papes contemporains, Benoît XV et Pie XI, sur les droits de la personne et le refus des totalitarismes, enseignement amplement diffusé par les évêques, le clergé, les organisations catholiques, a contribué à détourner la France du fascisme tout comme la force des traditions démocratiques et le poids des classes moyennes. Sans doute les radicaux qui, à la veille de la guerre, rendaient hommage à Pie XI lors de sa disparition $^{31}$, et qui souhaitaient un rassemblement des forces spirituelles contre le totalitarisme $e^{32}$, avaient-ils pris la juste mesure du rôle de l'Eglise catholique face aux périls qui menaçaient la démocratie française.

29 Cf. R. Rémond, Les catholiques.

30 François Valentin, dirigeant de la Jeunesse catholique de Nancy, est élu député en 1936, à moins de 27 ans. Il appartient à la Fédération républicaine.

31 Edouard Herriot à la Chambre, Jules Jeanneney au Sénat saluent le pape défenseur des droits de l'homme face au totalitarisme. La presse radicale socialiste a la même tonalité.

32 Cf. notre contribution sur la politique religieuse de Daladier dans: La question laïque XIXe-XXe siècle, Paris 1997. 